\title{
Lymphadenectomy during thoracoscopy: techniques and efficacy
}

\author{
Carlo Curcio, Dario Amore
}

Division of Thoracic Surgery, Monaldi Hospital, Naples, Italy

Correspondence to: Dario Amore. Division of Thoracic Surgery, Monaldi Hospital, Leonardo Bianchi street, 1, 80131 Naples, Italy.

Email: dario.amore@alice.it.

\begin{abstract}
Nowadays several studies have shown that, in the management of patients with early-stage lung cancer, video-assisted thoracoscopic surgery (VATS) lobectomy compared to open surgery is associated with many clinical short-term benefits, such as less post-operative pain and shorter hospital stay. Despite the advantages described earlier, some authors have expressed concern about the effectiveness of the procedure arguing that the lymph node harvest performed during VATS lobectomy is inferior to that performed through thoracotomy access. Experience from a multicentre database, with a more balanced number of VATS versus open patients, actually has shown that there is no difference in the efficacy of mediastinal lymph node dissection during lobectomy for lung cancer by thoracoscopy and thoracotomy. In expert hands the technique of VATS lymphadenectomy is the same as that performed by thoracotomy: instrumentation and tricks can change but not the oncologic principles. We believe that is necessary to follow a learning curve not only for VATS lobectomy but also for thoracoscopic lymph node dissection. However even experienced VATS surgeons should keep in mind that the metastatic lymphadenopathy with extracapsular lymph node spread requires great caution during dissection and that a preoperative plan for conversion to thoracotomy can be useful in this case.
\end{abstract}

Keywords: Video-assisted thoracoscopic surgery (VATS); lymphadenectomy; lung cancer

Received: 04 October 2017; Accepted: 12 October 2017; Published: 11 November 2017.

doi: 10.21037 /jovs.2017.10.08

View this article at: http://dx.doi.org/10.21037/jovs.2017.10.08

\section{Introduction}

Video-assisted thoracoscopic surgery (VATS) lobectomy is considered today a viable alternative to open thoracotomy for early-stage lung cancer treatment. Several studies have shown many short-term benefits of a minimally invasive approach compared to open surgery for major lung resection, such as reduced pain, shorter hospital stay, better cosmetic results and improved preservation of pulmonary function particularly for higher-risk patients $(1,2)$. Some reports, however, have expressed concerns that the lymph node harvest during VATS lobectomy is less than that achieved through thoracotomy $(3,4)$. The question about whether a VATS approach allows a complete lymph node dissection as a thoracotomy approach has raised doubts regarding the oncologic effectiveness of VATS lobectomy for non-small cell lung cancer (NSCLC) treatment.

\section{Efficacy}

Nowadays, regardless of open approach or minimally invasive approach, debate on the efficacy of mediastinal lymph node dissection versus mediastinal lymph node sampling, during pulmonary lobectomy for treatment of NSCLC, is still open. Several studies have shown that the mediastinal lymph node dissection allows a more accurate tumor staging, reducing the rates of local recurrence too (5). Other authors argue that there isn't the necessity of a complete lymph node dissection for pathologically proven stage I NSCLC by a systematic node sampling (6) and, according to some authors, lobe-specific mediastinal nodal evaluation appears acceptable in patients with early-stage NSCLC (7). Current practice guidelines by the National Comprehensive Cancer Network (NCCN) recommend to assess at least three mediastinal lymph node stations during lung cancer resections, according to the principles of 
surgical therapy in the treatment of NSCLC. However the implementation of mediastinal lymph node dissection versus mediastinal lymph node sampling is still controversial. The first procedure is associated with more accurate staging but it is not clear whether is associated with improved survival (8). Beyond the controversies surgeons anyway should not forget that getting an accurate lymph node staging is crucial to make adjuvant therapeutic decisions (6), keeping in mind at the same time potential complications from systematic mediastinal lymph node dissection such as: chylothorax and nerve injury (9). Regarding the concerns whether VATS, in comparison to thoracotomy, can achieve the same lymph node dissection efficacy in the treatment of lung cancer: we think that a minimally invasive approach isn't different from an open approach if during the steps of dissection the same oncologic principles are respected. Experienced VATS surgeons know that all lymph node stations can be resected by a thoracoscopic approach: the dissection procedure requires however specific tips and techniques (6). The thoracoscopic approach moreover, through use of the camera, allows an excellent visualization of the anatomical structures with magnification of the surgical field and in this way the lymph node stations can be more clearly identified during VATS lobectomy for lung cancer treatment $(10,11)$. Experience from a multicentre database has shown anyway that lymph node dissection during lobectomy for lung cancer by thoracoscopy and thoracotomy can be carried out with the same effectiveness, finding similar number of mediastinal lymph node stations and similar total number of lymph node stations resected with both approaches (8). Concerns about a VATS approach in the lymph node dissection, such as fewer nodes removed, less ability to remove level 7 nodes, upstaging of hilar and mediastinal nodes $(3,4,12)$, may be explained by a relative lack of experience with thoracoscopic approach (5). This means that with increasing experience in VATS, surgeons can achieve a nodal staging equivalent to thoracotomy approach. In literature however there is a growing amount of evidence that, for patients with clinical stage IA lung cancer, survival rates after lobectomy in VATS are not inferior to open thoracotomy $(13,14)$.

\section{Techniques}

To perform a proper lymph node dissection during VATS lobectomy, surgeons must bear in mind that a correct port placement can improve surgical field visualization and that the same placement may vary depending on the side of the chest, the body shape of the patient or his body mass index (BMI) value. The dissection procedure performed in VATS is facilitated by 30 degree thoracoscope that allows a clear identification of lymph node stations through a range of views, making easy the structures exposition due to the magnification of the surgical field (10). Many types of energy devices are available today and can make easier the lymph node dissection, although it's recommended to use them with care to avoid heat injury to vascular and nerve structures; it's undisputed however their usefulness to prevent bleeding that can obscure the field, slowing the dissection process due to poor viewing of the structures (15). Regarding mediastinal lymph node dissection, in some cases to improve the display of subcarinal and paraesophageal node stations it can be useful to retract the main bronchus anteriorly with a sponge stick and change the operating table orientation $(15,16)$. In the presence of hilar lymphadenopathy closely adherent to the pulmonary artery branches, it's advisable to perform careful dissection maneuvers and plan for a method of pulmonary artery proximal control via VATS or via conversion to thoracotomy.

\section{Our experience}

From our experience we have understood that, before lymph node dissection, it's important to know the patient's medical history asking specific questions, keeping in mind that preoperative chest CT scans may be the best way to get the imaging information needed to perform a safe and precision dissection. When we perform hilar lymph node dissection, we prefer to remove station 10 and 11 nodes before dividing the bronchovascular structures as this makes easier the dissection technique of pulmonary resection. In case of patients with lobar or sublobar lymph nodes disease and incomplete or fused fissure, we prefer, avoiding the risk of damage to lymph nodes, to perform the so called "tunnel technique": in this procedure dissection starts from the anterior part of the fissure, looking for a plane of cleavage between artery and parenchyma up to the posterior part of the fissure. In this way, after a complete opening of the incomplete o fused fissure with staplers, it's possible an optimal viewing of the bronchovascular structures and a complete intrafissural nodal dissection lowering risk of post-operative air leaks and unexpected intraoperative bleeding due to potential pulmonary artery injury. During VATS lobectomy for treatment of NSCLC we perform an accurate mediastinal lymph node staging: 


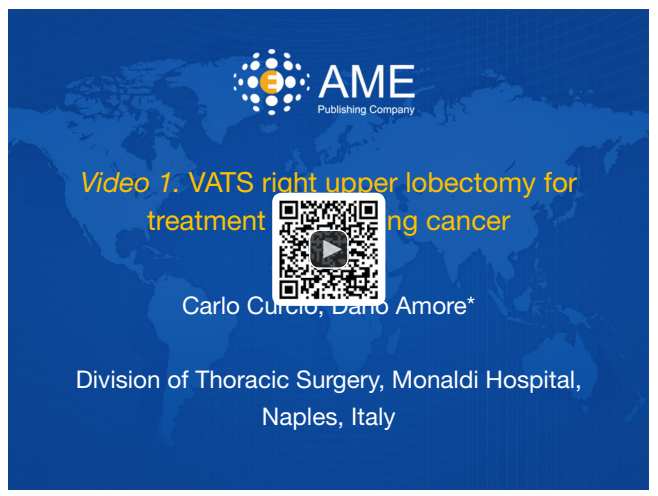

Figure 1 VATS right upper lobectomy for treatment of hilar lung cancer. The video shows, after transection of the right upper lobe vein, apical and anterior segmental arteries, hilar lymph node metastasis with extracapsular invasion. During bronchial dissection an intraoperative hemorrhage occurs due to injury of the pulmonary artery. To control the pulmonary artery proximal to the injured area, surgeons plan conversion to thoracotomy (17). VATS, video-assisted thoracoscopic surgery.

Available online: http://www.asvide.com/articles/1820

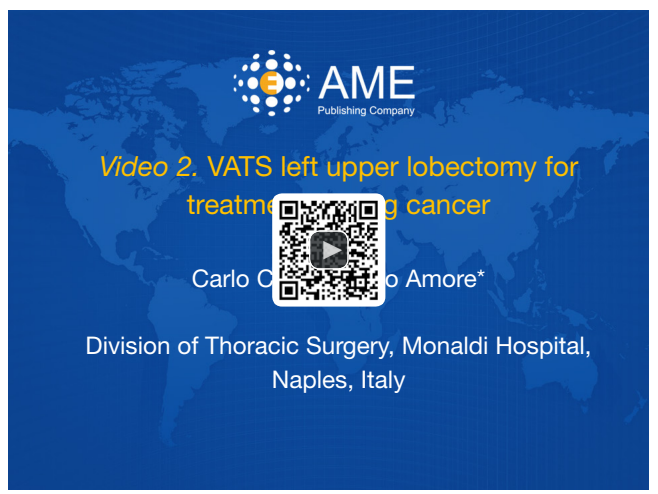

Figure 2 VATS left upper lobectomy for treatment of lung cancer. In this case, after transection of the superior pulmonary vein, apicoposterior, anterior and lingular segmental arteries, with dissection of the interlobar fissure, the exposure of the posterior segmental arteries, that shows a shorter course due to hilar lymphadenopathy, improves thanks to transection of the upper lobe bronchus (18). VATS, video-assisted thoracoscopic surgery. Available online: http://www.asvide.com/articles/1821

this procedure is crucial for selecting therapeutic strategies. According to our experience, developing specific skills and increasing use of minimally invasive technique, every nodal station can be dissected by a thoracoscopic approach but in our opinion nodes in station $4 \mathrm{~L}$ and $4 \mathrm{R}$ are more easily dissected after left and right upper lobectomy respectively. We think that surgeons should follow a learning curve not only for VATS lobectomy but also for lymphadenectomy. After the last stages of this curve it's possible to face major challenges during VATS lobectomy such as metastatic lymphadenopathy. In this case surgeons however should make a distinction between intracapsular and extracapsular lymph node involvement because an extracapsular lymph node spread is linked to a greater risk of vascular injury requiring sometimes conversion to open surgery (Figure 1). We believe that with the improvement of technical expertise in minimally invasive thoracic surgery, inflammatory adherent lymph nodes can be considered a relative contraindication to VATS lobectomy but in some cases nodal dissection tends to be time consuming or requires surgeons to change the sequential division of vein, arteries and bronchus planned (Figure 2).

\section{Acknowledgements}

None.

\section{Footnote}

Conflicts of Interest: The authors have no conflicts of interest to declare.

\section{References}

1. Berry MF, D'Amico TA, Onaitis MW, et al. Thoracoscopic approach to lobectomy for lung cancer does not compromise oncologic efficacy. Ann Thorac Surg 2014;98:197-202.

2. Paul S, Altorki NK, Sheng S, et al. Thoracoscopic lobectomy is associated with lower morbidity than open lobectomy: a propensity-matched analysis from the STS database. J Thorac Cardiovasc Surg 2010;139:366-78.

3. Boffa DJ, Kosinski AS, Paul S, et al. Lymph node evaluation by open or video-assisted approaches in 11,500 anatomic lung cancer resections. Ann Thorac Surg 2012;94:347-53; discussion 353.

4. Mathisen DJ. Is video-assisted thoracoscopic lobectomy inferior to open lobectomy oncologically? Ann Thorac Surg 2013;96:755-6.

5. Wang H, D'Amico TA. Efficacy of mediastinal lymph node dissection during thoracoscopic lobectomy. Ann Cardiothorac Surg 2012;1:27-32. 
6. Wright GM. VATS lobectomy lymph node management. Ann Cardiothorac Surg 2012;1:51-5.

7. Shapiro M, Kadakia S, Lim J, et al. Lobe-specific mediastinal nodal dissection is sufficient during lobectomy by video-assisted thoracic surgery or thoracotomy for early-stage lung cancer. Chest 2013;144:1615-21.

8. D'Amico TA, Niland J, Mamet R, et al. Efficacy of mediastinal lymph node dissection during lobectomy for lung cancer by thoracoscopy and thoracotomy. Ann Thorac Surg 2011;92:226-31; discussion 231-2.

9. Zhang W, Wei Y, Jiang H, et al. Thoracotomy is better than thoracoscopic lobectomy in the lymph node dissection of lung cancer: a systematic review and metaanalysis. World J Surg Oncol 2016;14:290.

10. Wang W, Yin W, Shao W, et al. Comparative study of systematic thoracoscopic lymphadenectomy and conventional thoracotomy in resectable non-small cell lung cancer. J Thorac Dis 2014;6:45-51.

11. Baisi A, Rizzi A, Raveglia F, et al. Video-assisted thoracic surgery is effective in systemic lymph node dissection. Eur J Cardiothorac Surg 2013;44:966.

12. Denlinger CE, Fernandez F, Meyers BF, et al. Lymph node evaluation in video-assisted thoracoscopic lobectomy

doi: $10.21037 /$ jovs.2017.10.08

Cite this article as: Curcio C, Amore D. Lymphadenectomy during thoracoscopy: techniques and efficacy. J Vis Surg 2017;3:167. versus lobectomy by thoracotomy. Ann Thorac Surg 2010;89:1730-5; discussion 1736.

13. Sugi K, Kaneda Y, Esato K. Video-assisted thoracoscopic lobectomy achieves a satisfactory long-term prognosis in patients with clinical stage IA lung cancer. World J Surg 2000;24:27-30; discussion 30-1.

14. Watanabe A, Mishina T, Ohori S, et al. Is video-assisted thoracoscopic surgery a feasible approach for clinical N0 and postoperatively pathological N2 non-small cell lung cancer? Eur J Cardiothorac Surg 2008;33:812-8.

15. Guido Guerrero W, Gonzalez-Rivas D, Hernandez Arenas LA, et al. Techniques and difficulties dealing with hilar and interlobar benign lymphadenopathy in uniportal VATS. J Vis Surg 2016;2:23.

16. Akiba T, Marushima H, Hirano K, et al. Thoracoscopic mediastinal lymph node dissection using an endoscopic spacer. Ann Thorac Cardiovasc Surg 2012;18:281-3.

17. Curcio C, Amore D. VATS right upper lobectomy for treatment of hilar lung cancer. Asvide 2017;4:501. Available online: http://www.asvide.com/articles/1820

18. Curcio C, Amore D. VATS left upper lobectomy for treatment of lung cancer. Asvide 2017;4:502. Available online: http://www.asvide.com/articles/1821 